\title{
AN AXISYMMETRIC BOUNDARY VALUE PROBLEM OF MIXED TYPE FOR A HALF-SPACE
}

\author{
by M. LOWENGRUB and I. N. SNEDDON $\dagger$ \\ (Received 2nd October, 1961)
}

\section{Introduction}

In problems in the mathematical theory of elasticity related to the symmetric deformation of an infinite elastic solid with an external crack we encounter the problem of determining an axisymmetric function $\phi(\rho, z)$ which is harmonic in the half-space $z>0$ and satisfies the mixed boundary conditions

$$
\begin{gathered}
\phi=0, \quad 0<\rho<1, \\
\frac{\partial \phi}{\partial z}=f(\rho), \quad \rho>1,
\end{gathered}
$$

on the plane boundary $z=0$, where it is assumed that $f(\rho)$ is continuously differentiable in $[1, \infty)$. Further $\phi \rightarrow 0$ as $\sqrt{ }\left(\rho^{2}+z^{2}\right) \rightarrow \infty$.

In this note we shall reduce the solution of this mixed boundary value problem to that of a simple pair of dual integral equations whose solution we derive by an elementary method in $\S 2$. By means of this solution we construct an integral representation of the function $\phi(\rho, z)$; its properties are discussed in $\S 3$. Finally, in $\S 4$, we derive the form of the solution in certain special cases.

\section{The Dual Integral Equations}

The integral

$$
\phi(\rho, z)=\int_{0}^{\infty} \xi^{-1} \psi(\xi) J_{0}(\xi \rho) e^{-\xi z} d \xi, \quad z \geqq 0,
$$

defines an axisymmetric harmonic function which tends to zero as

$$
\sqrt{ }\left(\rho^{2}+z^{2}\right) \rightarrow+\infty
$$

provided that the function $\psi(\xi)$ is such that the integrals (3) and

$$
\int_{0}^{\infty} \xi \psi(\xi) J_{0}(\xi \rho) e^{-\xi z} d \xi
$$

exist for $z>0$. If we substitute this expression into equations (1) and (2) we find

$\dagger$ The work described in this paper was done in the Department of Mathematics, Duke University, North Carolina, and was supported in part by the U.S. Air Force Office of Scientific Research, A.R.D.C., under contract AF 18(600)-1341. 
that the function $\psi(\xi)$ satisfies the dual integral equations

$$
\begin{aligned}
& G(\rho) \equiv \int_{0}^{\infty} \xi^{-1} \psi(\xi) J_{0}(\xi \rho) d \xi=0, \quad 0<\rho<1, \\
& F(\rho) \equiv \int_{0}^{\infty} \psi(\xi) J_{0}(\xi \rho) d \xi=f(\rho), \quad \rho>1 .
\end{aligned}
$$

This pair of equations is a special case of a pair considered by Williams (1) but it is of interest to note that the solution can be obtained by elementary methods similar to those employed in an earlier paper (2).

To solve the equations (4) and (5) we let

$$
\psi(\xi)=\xi \int_{1}^{\infty} \phi(t) \cos (\xi t) d t
$$

where $\phi(t)$ is such that the integral converges; in particular this implies the condition

$$
\lim _{t \rightarrow \infty} \phi(t)=0 .
$$

We first show that the form (6) automatically satisfies the first equation (4). Substituting from (6) into the expression for $G(\rho)$ and interchanging the order of integrations, we find that

$$
G(\rho)=\int_{1}^{\infty} \phi(t) d t \int_{0}^{\infty} J_{0}(\xi \rho) \cos (\xi t) d \xi
$$

It is well-known $(3$, p. 405$)$ that

so that

$$
\int_{0}^{\infty} J_{0}(\xi \rho) \cos (\xi t) d \xi= \begin{cases}0, & 0<\rho<t \\ \left(\rho^{2}-t^{2}\right)^{-\frac{t}{2}}, & \rho>t>0\end{cases}
$$

$$
G(\rho)=\left\{\begin{array}{ll}
0, & 0 \leqq \rho \leqq 1, \\
\int_{1}^{\rho} \frac{\phi(t) d t}{\sqrt{\left(\rho^{2}-t^{2}\right)}}, & \rho>1
\end{array} .\right.
$$

showing that equation (4) is satisfied.

By an integration by parts we find from (6) that we may write

$$
\psi(\xi)=-\phi(1) \sin \xi-\int_{1}^{\infty} \phi^{\prime}(t) \sin (\xi t) d t
$$

Substituting this expression for $\psi(\xi)$ into the equation defining $F(\rho)$ and interchanging the order of the integrations, we find that

$$
F(\rho)=-\phi(1) \int_{0}^{\infty} J_{0}(\xi \rho) \sin \xi d \xi-\int_{1}^{\infty} \phi^{\prime}(t) d t \int_{0}^{\infty} J_{0}(\xi \rho) \sin (\xi t) d \xi
$$

and making use of the standard result

$$
\int_{0}^{\infty} J_{0}(\xi \rho) \sin (\xi t) d \xi= \begin{cases}\left(t^{2}-\rho^{2}\right)^{-\frac{1}{2}}, & 0<\rho<t \\ 0, & \rho>t>0\end{cases}
$$


(3, p. 405) we find that when $0 \leqq \rho<1$

and that, when $\rho>1$,

$$
F(\rho)=-\frac{\phi(1)}{\sqrt{\left(1-\rho^{2}\right)}}-\int_{1}^{\infty} \frac{\phi^{\prime}(t) d t}{\sqrt{\left(t^{2}-\rho^{2}\right)}}
$$

$$
F(\rho)=-\int_{\rho}^{\infty} \frac{\phi^{\prime}(t) d t}{\sqrt{\left(t^{2}-\rho^{2}\right)}} .
$$

Using this last result we find that $\phi(t)$ must satisfy the integral equation

$$
\int_{\rho}^{\infty} \frac{\phi^{\prime}(t) d t}{\sqrt{ }\left(t^{2}-\rho^{2}\right)}=-f(\rho), \quad \rho>1 .
$$

This is an equation of Abel type and it is easily shown that, if $f(\rho)$ is continuously differentiable in $[1, \infty)$, it has the solution

$$
\phi^{\prime}(t)=\frac{2}{\pi} \frac{d}{d t} \int_{t}^{\infty} \frac{\rho f(\rho) d \rho}{\sqrt{\left(\rho^{2}-t^{2}\right)}}
$$

Integrating this equation and making use of the condition (7), we obtain the solution

$$
\phi(t)=\frac{2}{\pi} \int_{t}^{\infty} \frac{\rho f(\rho) d \rho}{\sqrt{ }\left(\rho^{2}-t^{2}\right)}
$$

The solution to our mixed boundary value problem is therefore given by equations (3), (6) and (14).

\section{An Integral Representation of the Solution}

If we substitute from equation (6) into equation (3), write the cosine in its exponential form and make use of the result

$$
\int_{0}^{\infty} J_{0}(\xi \rho) e^{-\lambda \xi} d \xi=\left(\rho^{2}+\lambda^{2}\right)^{-\frac{1}{2}}, \quad \mathscr{R}(\lambda)>0,
$$

we obtain the solution

$$
\phi(\rho, z)=\frac{1}{2} \int_{1}^{\infty}\left\{\frac{1}{\sqrt{\left[\rho^{2}+(z+i t)^{2}\right]}}+\frac{1}{\sqrt{ }\left[\rho^{2}+(z-i t)^{2}\right]}\right\} \phi(t) d t \ldots \ldots .
$$

We shall now follow the procedure adopted by Green in his solution of a similar boundary value problem (see (4), p. 172). We consider the function defined by equation (15) assuming that the function $\phi(t)$ is a real continuously differentiable function in $[1, \infty)$ for which the integral (15) converges.

Since the integrand on the right-hand side of equation (15) is a continuous function of $\rho, z$ and $t$ we may differentiate under the integral sign and verify that $\phi(\rho, z)$ is a solution of Laplace's equation.

We now introduce a function $\Phi(\rho, u)$ such that

$$
\frac{\partial \Phi}{\partial u}=\frac{1}{\sqrt{\left(\rho^{2}+u^{2}\right)}}
$$


We can then write equation (14) in the form

$$
\phi(\rho, z)=\frac{1}{2} i \int_{1}^{\infty}\left\{\frac{\partial \Phi(\rho, z+i t)}{\partial t}-\frac{\partial \Phi(\rho, z-i t)}{\partial t}\right\} \phi(t) d t
$$

and if we integrate this expression by the rule for integrating by parts, we obtain the relation

$$
\begin{aligned}
& \phi(\rho, z)=-\frac{1}{2} i \phi(1)[\Phi(\rho, z+i)-\Phi(\rho, z-i)] \\
& -\frac{1}{2} i \int_{1}^{\infty}[\Phi(\rho, z+i t)-\Phi(\rho, z-i t)] \phi^{\prime}(t) d t .
\end{aligned}
$$

The integrand occurring in the integral on the right-hand side of this equation is a continuous function of $\rho, z$ and $t$ so that we may differentiate under the integral sign to obtain the equation

$$
\begin{aligned}
& \frac{\partial \phi}{\partial z}=-\frac{1}{2} i \phi(1)\left\{\frac{1}{\sqrt{\left[\rho^{2}+(z+i)^{2}\right]}}-\frac{1}{\sqrt{\left[\rho^{2}+(z-i)^{2}\right]}}\right\} \\
& \quad-\frac{1}{2} i \int_{1}^{\infty}\left\{\frac{1}{\left.\sqrt{\left[\rho^{2}+(z+i t)^{2}\right]}-\frac{1}{\sqrt{\left[\rho^{2}+(z-i t)^{2}\right]}}\right\} \phi^{\prime}(t) d t .}\right.
\end{aligned}
$$

By using a method almost identical with that employed by Green we can show that $\partial \phi / \partial z$ is continuous for normal approach to the region $z=0$, $\rho>1$ as $z \rightarrow 0+$ and that

$$
\frac{\partial \phi}{\partial z} \rightarrow-\int_{\rho}^{\infty} \frac{\phi^{\prime}(t) d t}{\sqrt{\left(t^{2}-\rho^{2}\right)}}, \quad \text { as } z \rightarrow-0+.
$$

If we substitute this expression into equation (2) we obtain the integral equation (13) again, and the solution of this equation is given by equation (14). Hence we have shown that the solution to our mixed boundary value problem is given by equations (15) and (14).

\section{Special Cases}

In this section we shall derive the solutions of certain special problems which arise in physical applications.

Case 4.1. We begin by considering the case in which

$$
f(\rho)=\left\{\begin{array}{rr}
1, & 1<\rho<a \\
0, & \rho>a .
\end{array}\right.
$$

From equation (14) we find that

$$
\phi(t)= \begin{cases}\frac{2}{\pi} \sqrt{ }\left(a^{2}-t^{2}\right), & t \leqq a \\ 0, & t>a\end{cases}
$$


from which it follows immediately that

and that

$$
\psi(\xi)=\frac{2 \xi}{\pi} \int_{1}^{a} \sqrt{ }\left(a^{2}-t^{2}\right) \cos (\xi t) d t
$$

$$
\phi(\rho, z)=\frac{1}{\pi} \int_{1}^{a}\left\{\sqrt{\frac{a^{2}-t^{2}}{\rho^{2}+(z+i t)^{2}}}+\sqrt{\frac{a^{2}-t^{2}}{\rho^{2}+(z-i t)^{2}}}\right\} d t .
$$

From equation (12) we find that

$$
F(\rho)=\frac{2}{\pi} \int_{1}^{a} \frac{t d t}{\sqrt{\left[\left(a^{2}-t^{2}\right)\left(t^{2}-\rho^{2}\right)\right]}}-\frac{2}{\pi} \sqrt{\frac{a^{2}-1}{1-\rho^{2}}}, \quad 0 \leqq \rho<1
$$

and similarly from equation (9) we find that

$$
G(\rho)= \begin{cases}\frac{2}{\pi} \int_{1}^{a} \sqrt{\frac{a^{2}-t^{2}}{\rho^{2}-t^{2}}} d t, \quad \rho>a \\ \frac{2}{\pi} \int_{1}^{\rho} \sqrt{\frac{a^{2}-t^{2}}{\rho^{2}-t^{2}}} d t, \quad 1<\rho<a .\end{cases}
$$

The integral occurring in the expression for $F(\rho)$ is elementary. We find that

$$
F(\rho)=\frac{1}{2}+\frac{\alpha}{\pi}-\frac{2}{\pi} \sqrt{\frac{a^{2}-1}{1-\rho^{2}}}, \quad 0 \leqq \rho<1,
$$

where $\alpha$ is defined by the relations

$$
\sin \alpha=\frac{a^{2}+\rho^{2}-2}{a^{2}-\rho^{2}}, \quad-\frac{1}{2} \pi<\alpha<\frac{1}{2} \pi .
$$

The integrals in the expressions for $G(\rho)$ are easily expressed in terms of elliptic integrals:

$$
G(\rho)= \begin{cases}\frac{2 a}{\pi}[E(\rho / a)-E(\rho / a, \beta)], \quad 1<\rho<a, \\ \frac{2 \rho}{\pi}[E(a / \rho)-E(a / \rho, \gamma)]-\frac{2\left(\rho^{2}-a^{2}\right)}{\pi \rho}[K(a / \rho)-F(a / \rho, \gamma)], \quad \rho>a\end{cases}
$$

where $\beta=\sin ^{-1}(1 / \rho), \gamma=\sin ^{-1}(1 / a), 0<\beta<\frac{1}{2} \pi, 0<\gamma<\frac{1}{2} \pi$.

Case 4.2. We next consider the case in which

$$
f(\rho)=\rho^{-m}, \quad m>1 .
$$

From equation (14) we find that

$$
\phi(t)=\frac{t^{1-m} \Gamma\left(\frac{1}{2} m-\frac{1}{2}\right)}{\sqrt{\pi} \Gamma\left(\frac{1}{2} m\right)}, \quad(m>1),
$$


and from equation (6) that

$$
\psi(\xi)=\frac{\Gamma\left(\frac{1}{2} m-\frac{1}{2}\right)}{\sqrt{\pi} \Gamma\left(\frac{1}{2} m\right)} \xi \int_{1}^{\infty} t^{1-m} \cos (\xi t) d t,
$$

which is equivalent to

$$
\psi(\xi)=\frac{\Gamma\left(\frac{1}{2} m-\frac{1}{2}\right)}{\sqrt{\pi} \Gamma\left(\frac{1}{2} m\right)} \xi^{m-1} \int_{\xi}^{\infty} \lambda^{1-m} \cos \lambda d \lambda
$$

Equations (15) and (22) then give the expression

$\phi(\rho, z)=\frac{\Gamma\left(\frac{1}{2} m-\frac{1}{2}\right)}{2 \sqrt{\pi} \Gamma\left(\frac{1}{2} m\right)} \int_{1}^{\infty}\left\{\frac{1}{\sqrt{\left[\rho^{2}+(z+i t)^{2}\right]}}+\frac{1}{\sqrt{\left[\rho^{2}+(z-i t)^{2}\right]}}\right\} t^{1-m} d t, \ldots$

for the harmonic function $\phi$.

From equation (12) we find that

$$
F(\rho)=\frac{\Gamma\left(\frac{1}{2} m-\frac{1}{2}\right)}{\sqrt{\pi} \Gamma\left(\frac{1}{2} m\right)}\left\{(m-1) \int_{1}^{\infty} t^{-m}\left(t^{2}-\rho^{2}\right)^{-\frac{1}{2}} d t-\left(1-\rho^{2}\right)^{-\frac{1}{2}}\right\}, \quad 0 \leqq \rho<1 .
$$

Expanding the integrand in powers of $\rho^{2} t^{-2}$ and integrating term by term we obtain the expression

$F(\rho)=\frac{\Gamma\left(\frac{1}{2} m+\frac{1}{2}\right)}{\sqrt{\pi} \Gamma\left(\frac{1}{2} m+1\right)}{ }_{2} F_{1}\left(\frac{1}{2}, \frac{1}{2} m ; \frac{1}{2} m+1 ; \rho^{2}\right)-\frac{\Gamma\left(\frac{1}{2} m-\frac{1}{2}\right)}{\sqrt{\pi} \Gamma\left(\frac{1}{2} m\right)}\left(1-\rho^{2}\right)^{-\frac{1}{2}}, 0 \leqq \rho<1$.

Similarly from equation (9) we find that, when $\rho>1$,

$$
G(\rho)=\frac{\Gamma\left(\frac{1}{2} m-\frac{1}{2}\right)}{\sqrt{\pi} \Gamma\left(\frac{1}{2} m\right)} \int_{1}^{\rho} \frac{t^{1-m} d t}{\sqrt{ }\left(\rho^{2}-t^{2}\right)}
$$

a result which can be written in the form

$$
G(\rho)=\frac{\Gamma\left(\frac{1}{2} m-\frac{1}{2}\right)}{2 \sqrt{\pi} \Gamma\left(\frac{1}{2} m\right)} \rho^{-\frac{1}{2} m} B_{r}\left(\frac{1}{2}, 1-\frac{1}{2} m\right), \quad r=1-\rho^{-2}, \quad m>1,
$$

where $B_{r}\left(\frac{1}{2}, 1-\frac{1}{2} m\right)$ denotes the incomplete beta function

$$
\int_{0}^{r} u^{-\frac{1}{2}}(1-u)^{-\frac{1}{2} m} d u \text {. }
$$

In (5) we can find tables of the function $I_{x}(p, q)=B_{x}(p, q) / B(p, q)$ which can be used in the computation of $G(\rho)$.

Case 4.3. These results take a simpler form in the case in which $m=2$, i.e. when $f(\rho)=\rho^{-2}$. In this instance we have, from equation (14),

$$
\phi(t)=t^{-1}
$$

from which it follows that

$$
\psi(\xi)=-\xi \operatorname{Ci}(\xi)
$$


where, in the usual notation, $\mathrm{Ci}(\xi)$ denotes the cosine integral

$$
\operatorname{Ci}(\xi)=-\int_{\xi}^{\infty} u^{-1} \cos u d u
$$

Equation (3) then yields the form

$$
\phi(\rho, z)=-\int_{0}^{\infty} \operatorname{Ci}(\xi) J_{0}(\xi \rho) e^{-\xi z} d \xi,
$$

for the harmonic function $\phi$, while equation (15) gives the form

$$
\phi(\rho, z)=\frac{1}{2} \int_{1}^{\infty}\left\{\frac{1}{\sqrt{\left[\rho^{2}+(z+i t)^{2}\right]}}+\frac{1}{\sqrt{ }\left[\rho^{2}+(z-i t)^{2}\right]}\right\} \frac{d t}{t} .
$$

From equation (12) we find that

$$
F(\rho)=\int_{1}^{\infty} \frac{d t}{t^{2} \sqrt{ }\left(t^{2}-\rho^{2}\right)}, \quad 0<\rho<1
$$

and from equation (9) that

$$
G(\rho)=\int_{1}^{\rho} \frac{d t}{t \sqrt{ }\left(\rho^{2}-t^{2}\right)}, \quad \rho>1 .
$$

The integrations are elementary; carrying them out we obtain the expressions

$$
\begin{aligned}
& F(\rho)=\frac{1}{\rho^{2}}\left[1-\sqrt{ }\left(1-\rho^{2}\right)\right]-\frac{1}{\sqrt{ }\left(1-\rho^{2}\right)^{\prime}}, \quad 0<\rho<1 \\
& G(\rho)=\frac{1}{\rho} \log \left[\rho+\sqrt{ }\left(\rho^{2}-1\right)\right], \quad \rho>1 . \ldots \ldots \ldots \ldots \ldots \ldots \ldots \ldots
\end{aligned}
$$

Case 4.4. We now consider the case in which

$$
f(\rho)=\frac{\delta(\rho-c)}{2 \pi \rho}, \quad c>1
$$

$\delta(z)$ denoting the Dirac delta function of argument $z$. From equation (14) we find that

$$
\phi(t)=\left\{\begin{array}{lr}
\pi^{-2}\left(c^{2}-t^{2}\right)^{-\frac{1}{2}}, & 0 \leqq t<c, \\
0, & t>c,
\end{array}\right.
$$

and hence from equation (6) we obtain the expression

$$
\psi(\xi)=\frac{\xi}{\pi^{2}} \int_{1}^{c} \frac{\cos (\xi t) d t}{\sqrt{\left(c^{2}-t^{2}\right)}}
$$

From equation (9) we see that

$$
G(\rho)=\frac{1}{\pi^{2}} \int_{1}^{\lambda} \frac{d t}{\sqrt{\left[\left(c^{2}-t^{2}\right)\left(\rho^{2}-t^{2}\right)\right]}}
$$

where $\lambda=\rho$ if $1<\rho<c$ and $\lambda=c$ if $\rho>c$; carrying out the integrations we 
find that

$$
G(\rho)= \begin{cases}\frac{1}{\pi^{2} c}[K(\rho / c)-F(\rho / c, \beta)], & 1<\rho<c ; \\ \frac{1}{\pi^{2} \rho}[K(c / \rho)-F(c / \rho, \delta)], & \rho>c,\end{cases}
$$

where $\beta$ is defined as in equation (21) above and $\delta$ is defined by the relations $\sin \delta=1 / c, 0<\delta<\frac{1}{2} \pi$.

Case 4.5. Finally we consider the case in which $f(\rho)$ is defined by an integral expression of the form

$$
f(\rho)=\int_{0}^{\infty} \xi \bar{f}(\xi) J_{0}(\xi \rho) d \xi
$$

where the function $f(\xi)$ is the Hankel transform of order zero of $f(\rho)$. From equation (14) we derive the result

$$
\phi(t)=\frac{2}{\pi} \int_{0}^{\infty} \xi \bar{f}(\xi) d \xi \int_{t}^{\infty} \frac{\rho J_{0}(\xi \rho)}{\sqrt{\left(\rho^{2}-t^{2}\right)}} d \rho .
$$

Carrying out the $\rho$-integration we obtain the formula

$$
\phi(t)=\frac{2}{\pi} \int_{0}^{\infty} \bar{f}(\xi) \cos (\xi t) d \xi
$$

\section{REFERENCES}

(1) W. E. Williams, The solution of certain dual integral equations, Proc. Edinburgh Math. Soc., 12 (Ser. ii) (1961), 213.

(2) I. N. Sneddon, The elementary solution of dual integral equations, Proc. Glasgow Math. Assoc., 4 (1960), 108.

(3) G. N. Watson, A Treatise on the Theory of Bessel Functions, 2nd edn. (University Press, Cambridge, 1944).

(4) A. E. Green and W. Zerna, Theoretical Elasticity (Clarendon Press, Oxford, 1954).

(5) K. Pearson, Editor, Tables of the Incomplete Beta Function (University Press, Cambridge, 1932).

North Carolina State College

RALEIGH

North Carolina
THE UNIVERSITY

GlasGow 